Wieviel Erde braucht der Mensch, wieviel Materialbesitz und wieviel Haben kann er ersetzen durch Sein? Wir unterscheiden bisher bleibendes Eigentum vom Besitz.

Auf die Dauer gesehen ist alles Sichtbare Besitz, auch unser Körper, dessen Baustoffe wir der Umwelt entliehen haben und die wir später an sie zurückgeben. Die Neuorientierung nach dem wahren Lebensbedarf kann das alte Projekt Menschwerdung neu beflügeln und mit ihr eine frische Pädagogik.

(Nikolaus von Kaisenberg)

\title{
Veränderung \\ ist eine gute Schule
}

den Wandel am Wesentlichen ausrichten

\section{Nikolaus von Kaisenberg}


Nikolaus von Kaisenberg stellt sich in diesem Beitrag die Frage, wie weit sich Schule vom eigentlichen Leben entfernt hat, und er erörtert, inwieweit diese gesellschaftliche Veranstaltung den natürlichen Anlagen von Entwicklung gerecht werden und dazu beitragen kann, die individuellen und die gemeinschaftlichen Potenziale der Menschen zur Entfaltung zu bringen. Das traditionelle Schulhaus steht laut Kaisenberg im Widerspruch zu einer entwicklungsoffenen Pädagogik und er sucht danach, wie Raumbildung und Lebensprozess vermehrt in einen Dialog treten können. Eine Orientierung am wahren Lebensbedarf kann aus seiner Sicht das alte Projekt Menschwerdung neu beflügeln und damit gleichzeitig Pädagogik und Schulbau wandeln.

\section{Neues in die Welt bringen}

Wie kommt das Neue in die Welt, wenn es nach den Regeln des Alten Vorbereitung findet? Erziehung und Schule sind Lebensfelder, in denen Veränderung kontrolliert angelegt und optimiert werden soll. Es muss etwas geschehen, aber passieren darf nichts, so heißt es. Dieser Beitrag geht vom Widerstreit der Kräfte aus, der am Bildungswesen und damit an den betroffenen Menschen rüttelt. Und er entwirft ein Bild zum Einklang von Leben und Lernen.

\section{Leben ist Ort ständiger Veränderung}

Leben ist Ort ständiger Veränderung. Veränderungsimpulse kommen von außen auf uns zu, und auch in uns selbst richten sie sich auf, suchen Raum und Gehör. Manchmal ist die Wahrnehmungsbrücke zur Umgebungswelt oder zu uns selbst durch Gedankengebäude so geschwächt, dass wir zurückbleiben in Stagnation. Die Entwicklung geht indessen weiter, und wir bleiben mit ihr über ein elastisches Band verbunden. Mit zunehmender Entfernung spannt sich das Band immer mehr. Und sobald wir unser Beharren loslassen, wirft es uns weit nach vorne, in eine neue Situation. Darum geschieht Entwicklung in Sprüngen, in Stufen der Veränderung, oder auch in Transformationen, also durch Bereinigung und Neubeginn. So kann eine verwandelte Lebenshaltung eine neue Lernhaltung und Lernbereitschaft mit sich bringen. Was wir von außen wahrnehmen, aus der Natur, können wir innen nicht immer hören. Der Umgang mit der Erde ist auch der Umgang mit uns selbst, dessen Art und Weise wir kaum wahrhaben wollen, auch wenn er Konflikte mit 
dem eigenen Körper und im Sozialen mit sich bringt. Umgekehrt lassen sich unsere inneren Veränderungsimpulse oft nicht in der sichtbaren Welt manifestieren, weil uns auch dort vorgefasste Vorstellungen und Absichten im Weg stehen. Nun haben wir aktuell allen Anlass, diese beiden Welten des äußeren und inneren Erlebens mehr zum Dialog und zur Deckung zu bringen. Über Jahrtausende fühlte sich die Menschheit bedroht von der wilden Natur der Erde, von peitschendem Regen, von Frost oder von Wüstendürre. Nun hat sich innerhalb kurzer Zeit das Blatt gewendet - schutzbedürftig ist nun der Planet. Und wir suchen inn nicht nur als Objekt, sondern nun als ebenbürtigen Lebenspartner zu sehen, inn als eigene Ganzheit und Organismus zu verstehen und dabei auch zu neuem Selbstverständnis zu kommen. Wir spüren darin einen neuen Anfang.

\section{Schule und Leben im Widerspruch}

Wenige Lebensfelder sind so konsequent von Paradigmen begrenzt, wie das Feld der Schule: „Eltern gestalten das Leben, Schule organisiert das Lernen", so erklärt sich unausgesprochen unser Schulsystem. Die Begrenztheit eines Paradigmas ist uns nicht bewusst, das gehört zu seinem Wesen. Es ist auch weniger der Fokus einer Denkungsart, der hinderlich ist, sondern die anhaltende Abtrennung von den ausgegrenzten Lebensinhalten. Schule steht als geschlossenes System nicht allein da. Die zunehmende Selbstbezüglichkeit gilt auch für andere Teilsysteme der modernen Gesellschaft, zum Beispiel für das Gesundheitswesen, für Wirtschaft, Wissenschaft und Kirche. „Pisa“ als normative Schulleistungsstudie mit internationaler Geltung und "Bologna" als akademisches Raster für homogene Lernstrukturen europäischer Studiengänge haben Selfishness als Eigenlegitimation der Einrichtungen verstärkt und den Lebensbezug gemindert (Sloterdijk 2009). Alles was Schule und Hochschule weiß, weiß und begründet sie aus sich heraus. Wie bei der Kanalisierung eines Flusses werden die Berührungsflächen zum Umgebungsfeld der Schulen immer kleiner; einzelne Flussschleifen werden abgetrennt. Sie treten zunehmend in Form von Reparaturbetrieben wieder auf, u. a. als Förderschulen, therapeutische Einrichtungen, pädagogische Ambulanzen, Familienorientierungszentren und Willkommensstätten für Schulverweiger·innen. Die genannten Einrichtungen stabilisieren und legitimieren sich wiederum in wachsender Ausdifferenzierung. Zwischen heutigen Schulsystemen gibt es Unterschiede: Diese sind eher gradueller Natur, solange 
Lernziele über Lebensziele gestellt werden. Diese heißen Entfaltung und Erfüllung. So fehlt dem Bildungswesen eine Renaturierung, die es durch Optimierung des eingeschlagenen Weges sucht. Scheibchenweise soll Schule weiter verändert werden durch bessere Werkzeuge, bessere Gebäude und mehr Entspannungsangebote für Schüler.innen und Lehrkräfte, deren Situation von Widersprüchen und Anspannung geprägt ist.
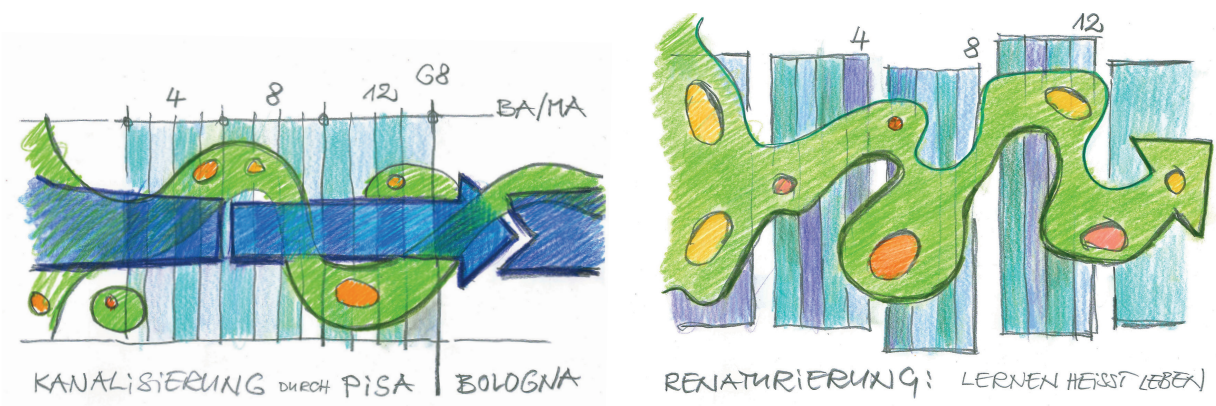

ABB 01 Kanalisierung des Lernens @ 2020 Nikolaus Kaisenberg (links); Renaturierung des Lebens @ 2020 Nikolaus Kaisenberg (rechts)

Schule ist ein Ganztagsgeschehen geworden. Weil der Weg dahin in einzelnen Schritten von graduellem Charakter geschah, wird leicht übersehen, dass inzwischen ein prinzipieller Wandel nachzuvollziehen ist. Schule ist Lebensraum geworden - für die Lernenden, aber auch zunehmend für die Lehrenden. Darum stehen Schulbauten nun zur Erneuerung an. Und weil dabei über den sinnvollen Einsatz von Finanzmitteln in Milliardenhöhe entschieden wird, werden Schulbauten zunehmend von verschiedenen Seiten beleuchtet und befragt. Dabei zeigt sich öfter, dass "der Kittel zwickt": Die Pädagogik hat sich den Einschränkungen der überkommenen Gebäude und Zwecke angepasst. Wenn das Kleid zu eng ist, neigt die Erneuerung von Lernformen zu Tippelschritten, wo inzwischen eine großzügigere Bewegung angebracht wäre. Also baut man Schulen heute größer. Aber ist das alles, ist das die ausreichende Antwort zur Erneuerung von Schule?

Wer die meiste Zeit der Woche in der Schule verbringt, hört nicht mehr gerne die alte Sentenz, Schule sei Vorbereitung aufs Leben. Nein, Schule ist schon das Leben. Und Schüler.innen wollen dieses Leben lieben können. Sie spüren jede Form von Simulation, wagen kaum ihr zu widersprechen. Aber sie ziehen das Echte vor, also das Leben, das für sie unverwechselbar sein möchte, einmalig, erstmalig und kostbar. 


\section{lassen wir uns genug herausfordern?}

Wo für uns Erziehung und Schule abgeschlossen waren - kam da jemals eine Person in ihrer unverstellten Menschlichkeit zur Erscheinung? Leuchtet heute beim Schulabschluss das Ganze, ungetrübte Potenzial durch die jungen Menschen? Was ist geschehen während der Schulzeit, und was kann geschehen, damit Menschen sich zum eigenen Wohle und zum Gedeihen der Gesellschaft mehr an ihre Begabungen annähern, wenn Unterschiede mehr gelebt werden können als die Gleichförmigkeit?
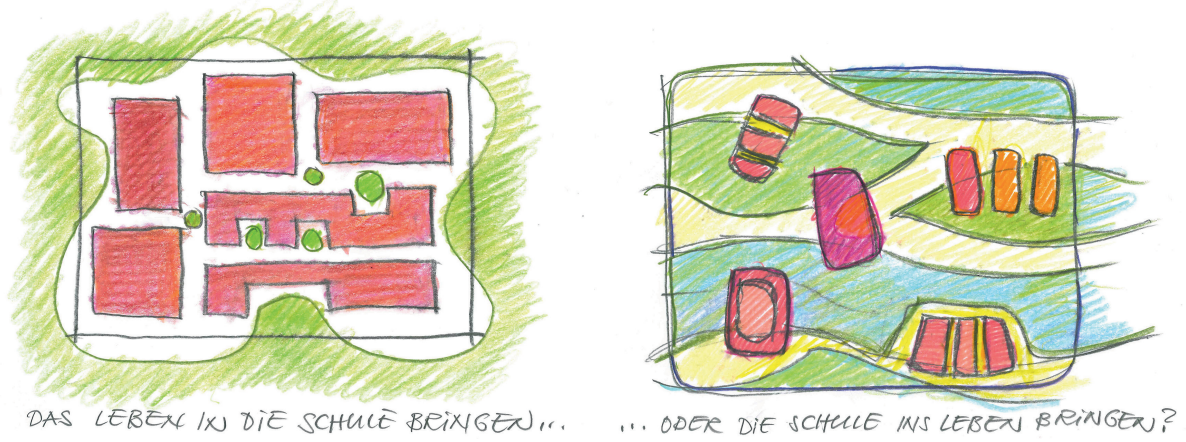

ABB 02 Das Leben in Resträume bringen @ 2020 Nikolaus Kaisenberg (links); Lernformen mit Leben in Einklang bringen @ 2020 Nikolaus Kaisenberg (rechts)

Das Leben, das bisher nach den Schulstunden stattfand, soll nun in den Schultag eingefügt werden, wo der Unterrichtsplan dafür Lücken bietet. Wie auch das Leben in Restflächen der Gebäude hineingebracht werden soll. Können wir diesem Ablauf eine Wendung geben? Können wir das Verhältnis von Lernen und Leben umwenden und einen Lebensstrom entwickeln, in den sich verschiedene Lernformen geschmeidig einfügen? Und welche Perspektive nehmen wir dabei ein? Hierzu zwei Blickwinkel aus Sicht der Betroffenen.

\section{sind Kinder}

\section{wie Pflänzchen und Kameras?}

Vergleichen wir Kinder mit Pflänzchen: sie kommen selbst zur Entfaltung, wenn man ihnen den Nährboden gibt, Schutzraum und Licht. Wir brauchen nicht an ihnen zu zupfen, sie wachsen wie von selbst. Und nach den ersten Blättern, die das Pflänzchen treibt, können wir uns ge- 
dulden und auf die Ausformung der nächsten Blätter warten. Nach und nach wächst das Gebilde hinein in seinen angestammten Raum, treibt Knospen, Blüten und zeigt Früchte. Für Kinder ist diese Veränderung ein natürlicher Prozess, dem sie sich anvertrauen wie dem Wachstum ihres Körpers. Dieser verändert sich alle paar Monate dramatisch. Er verliert sogar Zähne, die Proportion und die Stimmhöhe. Trotzdem geben Kinder sich diesem Strom der Entfaltung hin, als ob sie ihn schon öfter erlebt hätten. Sie möchten ihm auch weiter folgen und zum Abschluss der Schulzeit das Potenzial ihres Menschseins zur Erscheinung bringen. Was hält sie auf? Warum zeigen sie Seiten, die wir nicht erwartet hatten? Gehören sie dem Entfaltungsinhalt an oder spiegeln sich Eigenschaften, die sie in ihrem kurzen Zusammenleben mit uns erworben haben?

Wir alle kennen Kinder, die lauter agieren. Als wollten sie sich vergewissern, dass man sie hört und dass sie sich selbst hören in ihrem Anliegen, das sie zwar nicht kennen, nach dessen Gesicht sie sich jedoch sehnen und es gleichzeitig fürchten. Denn sie ahnen, dass es einen neuen Lebensentwurf entpuppen und Konsequenzen fordern könnte, die der gesellschaftlichen Mechanik widersprechen. Darum schwanken sie zwischen Anpassung und Protest. Sie wissen nicht, welcher Seite sie trauen sollen. Das Schwanken zwischen inneren und äußeren Stimmen ist wie ein Wackelkontakt, es macht sie zu Recht rappelig mitsamt ihrem Umfeld, in das sie hineinagieren. Diese jungen Menschen sind für uns genauso kostbar wie alle anderen, wir wollen ihre Botschaften hören und sie für unsere gewinnen - und für sich selbst.

Eine andere Gruppe Jugendlicher, die uns auch fehlt, hat sich weit in sich zurückgezogen, viel zu weit, unauffindbar fast für uns und für sich selbst. Sie sind einfach sprachlos. „So hatten wir uns das nicht ausgemalt", scheinen sie stumm zu sagen. Sie verbringen ihre Zeit mit Warten, mit Sollen und Müssen - und nicht mit der freien Entfaltung ihres Lebensplanes, zu dem sie kaum Kontakt finden. Sie fühlen sich mundtot gemacht von allem was schon fertig vorbereitet vor ihnen liegt. „Was sollen wir hier, alles ist fertig und wird von den Erwachsenen in jedem Detail verteidigt, obwohl angeblich Wandel angesagt ist". Jeder zaghafte Eingriff in ihr Leben verhallt wie in Watte, jeder Ausdruck wird vom Umfeld zurückgefedert wie ein Eindruck in Schaumstoff. Beide Gruppen sind verstört über die Widersprüchlichkeit von Zielen, die Erwachsene miteinander zu vereinen suchen, z. B. regionaler Konsum und globaler Frieden. Das Befolgen von Regeln verursacht auch heute nicht weniger Schäden an Menschen als ihre Verweigerung. Das Ergebnis zeigt traumatischen Charakter, obwohl es keinen ursächlichen Unfall, kein einschneidendes Unglück gab. Kaum einer kann sagen, wo 
das Elend der Betroffenen begann. Im schulischen Separierungsprozess ist nicht mehr zu unterscheiden, ob Anpassung oder Verweigerung Ursache der Aussonderung sind. Denn die habituellen Folgen sind ähnlich. Die Erwachsenen empfinden jede Abweichung als Störung und Sprengung ihres Konzeptes, das den Beteiligten Form und Verlässlichkeit bieten soll und zum Gegenteil führt. Vielleicht ist auch genau das die Botschaft - Sprengung der Konzepte zuzulassen, dem Entfaltungsstrom Raum geben? Ihm steht ein anderer Strom entgegen, ein Gegenstrom:

Ist es vielleicht so, dass Kinder wie Kameras alles in sich aufnehmen und sie es später auf vielschichtigen Wegen wiedergeben? Und so, wie die Kamera nicht nur das gewählte Motiv des Fotografen wiedergibt, sondern auch den ganzen Hintergrund, so nehmen Schulkinder nicht nur den Unterrichtsstoff auf, der momentan im Fokus ihrer Aufmerksamkeit stehen soll, sondern auch das ganze Lebensumfeld mit allen Einzelheiten. Sie werden Abbild einer ganzen Organisation, in der sie leben, sei es die Familie oder die Schule.

Neben dem pädagogischen Konzept spiegeln sie auch die Lebensform der Schulgemeinschaft. Und wie ihr Körper die Baustoffe und die Bauform der Gebäude aufnimmt, so sind sie seelisch empfänglich für die sozialen Lebensumstände, etwa für den Grad der Gemeinschaftlichkeit oder für die Kräfte der Selbst- oder Fremdbestimmtheit. Sie haben auch ein Gespür für die vorprägende oder öffnende Kraft von Konzepten. Heißt das pädagogische Konzept: „Wissen was passiert, bevor es passiert" (Verlängerung des in der Vergangenheit Erlernten in die Zukunft) oder heißt es, einen gemeinsamen Entwicklungsweg gehen zu können (Schüler-innen, Lehrkräfte und Eltern wenden sich dem zu Erlernenden zu, das aus dem Moment kommt).

Strom und Gegenstrom gehören zu den natürlichen Kräften, die einer Biografie unverwechselbare Gestalt und eigene Kraft geben können, wenn sie einigermaßen ausgewogen sind, wenn der Entfaltungsstrom dem Gegenstrom nicht unterliegt, sondern wachsen und sich gerade daran erschließen kann. Die Temperamente reagieren unterschiedlich und sind auch auf verschiedene Weise empfänglich für Begleitungen. Die Temperamente des Cholerischen und Sanguinischen reagieren eher elastisch auf Eindrücke und Vorgaben, sie federn sie ab oder antworten auf Kraft von außen mit eigenen Impulsen von innen. Während die phlegmatisch-melancholische Lebenshaltung, eher plastische Qualität zeigt, indem sie Ereignisse bereitwilliger aufnimmt, und dann auch eher unverändert beibehält (Smit 1989, 19f). 


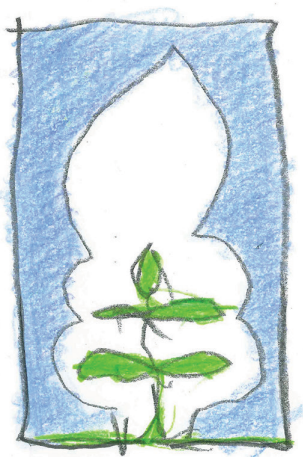

STRON

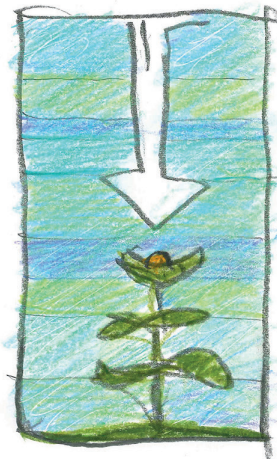

GEGENSTREOM

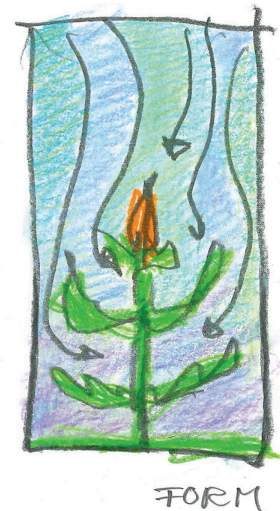

FORM

ABB 03 Strom, Gegenstrom, individueller Entfaltungsraum @ 2020 Nikolaus Kaisenberg

\section{Schule am ganzen Tag. Prägungen und Wandlungen}

Die Prägungen, die auf die Schüler $\cdot$ innen wirken, sind umfassender, als es eine Betrachtung vermuten lässt, die allein von den baulichen Anlagen her schaut. Alle Einprägungen werden zu latenten Ausprägungen. Das Ortsgedächtnis erinnert immer wieder an die Örtlichkeit von Schlüsselerlebnissen. Den Satz des Pythagoras hat Franziska zum ersten Mal im grünen Klassenzimmer kapiert. Der Flur, auf den Franz öfter rausflog, roch nach welkenden Pflanzen. Prägungen rühren auch vom Unterrichtsstoff her, von den Mitschüler-innen, von den Lehrenden sowie auch vom Zeitpunkt im Tages- oder Wochenlauf, sowie von den Jahreszeiten und Festlichkeiten.

Wo Schule ein Bestandteil sozialer Ordnung war, da wird sie nun selbst Ausgangspunkt einer Lebensordnung, sie könnte Quellpunkt neuer Lebensentwürfe werden. Ihre neue Rolle ist noch überlagert von den morphogenetischen Feldern ihrer Herkunft - von der Klosterschule und von der Militärschule. Schule neigt daher zur Formung, zur Aufprägung einheitlicher Form. Wo Form Bestandteil der Bestrebungen ist, will - als Gegenpart - auch Auflösung überkommener Form zum Inhalt und Werkzeug des Lernens werden. Darum liegt im Fokus des Beitrages der Blick auf das Verständnis von Schutz und Freiheit als Gegensatzpaar. Den Kindern Raum geben - Schutzraum und Freiräume, den Lehrer.innen und Eltern Raum geben für Veränderung und dafür Phasen zuzulassen zur Auflösung einer starr gewordenen Struktur und zugunsten einer Neuordnung, die den Lebensanliegen der Menschen folgt, darin liegt die Herausforderung unserer Zeit.

Das Schulhaus, mit dem Architekturschaffenden etwas Fertiges verbinden, steht im Widerspruch zu einer entwicklungsoffenen Pädagogik. 
Wie können Raumbildung und Lebensprozess mehr aufeinander eingehen. Gestaltungsstarke Architekt.innen wie Hans Scharoun haben immer wieder am herkömmlichen Typus Schule gerüttelt. Er versucht die pädagogischen Ziele zu erweitern, indem er "Schul-Wohnungen" anlegt, mit denen er über das Konzept der Wissensvermittlung hinausgeht und in der Sozialform "familiären Lernens auch Vorgänge des Wachstums und der Entwicklung" veranlagt. Er beruft sich dabei auf Hugo Häring, "welcher das Bauwerk ,ein Organ des Lebens' nannte, weil er die Welt und ihre Einzelheiten vom Geschehen her begreift" (Janofske 1984, 5). So zeigt Scharoun mit der Planung des Geschwister-Scholl-Gymnasiums in Lünen (1956) eine dörfliche Gemeinschaftsbildung mit einer nischenreichen inneren Straße, mit Seitengassen und mit Außenklassen (vgl. Zinner $\rightarrow \mathrm{nAB} 220$, 8). Das Schulhaus sucht Anbindung nach draußen in die Stadtstruktur; gleichzeitig spiegelt es das Konzept der Abtrennung vom Leben. Worüber bis heute in den Schulen nur berichtet wird, das wird dort in Werkstätten und Labors simuliert, aber bisher nicht umfänglich gelebt (Kaisenberg 2017).

\section{Lichtblicke}

Einzelne Schulen wagen die funktionale Entgrenzung von Schule und Stadtleben, von Schule und Landleben, sowie von der Natur. Sie kooperieren mit einem Bauernhof oder machen diesen zum Bestandteil ihrer Schule. Sie übernehmen ein Zirkusprojekt und wandern damit durch die Stadtteile, oder sie bewirtschaften gemeinsam mit Nachbar.innen öffentliche Grünanlagen. Was immer diese Schulen tun, sie vermitteln der nächsten Generation Schlüsselerlebnisse der Selbstwirksamkeit und der Lebenstüchtigkeit - in einer Situation globaler Krise. Neben dem Schulabschluss schauen Eltern und Lehrer-innen auf Schulanschluss, auf Zusammenschluss bisher getrennter Lebensbereiche und Biografie-Abschnitte. Das Curriculum bislang separierter Unterrichtsfächer möchte aufgehen in einen fachbergreifenden Projektunterricht. Dies wird gefördert durch Vergrößerung von zwei Berührungsflächen. Die eine betrifft die Vergrößerung der Selbstbegegnung, die andere liegt zwischen Schule und Umgebungswelt, die durch kulturelle Renaturierung des Lebensflusses geschehen kann. Schule wächst als Ort mitten im Leben. 


\section{Verbindungen}

$\rightarrow \mathrm{nAB} 101$ aus sich heraus lernen

$\rightarrow$ nAB141 personalisiert lernen

$\rightarrow$ nAB142 Realprojekte

$\rightarrow$ nAB220 von Dorfschule zu Schuldorf

\section{Literatur}

Janofske, Eckehard (1984): Architektur-Räume, Idee und Gestalt bei Hans Scharoun. Braunschweig: Vieweg.

Kaisenberg, Nikolaus von (2017): Selber machen - Idee und Praxis einer entwicklungsoffenen Architektur. In: Mensch und Architektur [Heft 91/92: Partizipation. Soziale Prozesse in der Architekturgestaltung] 7/2017, 22-26.

Sloterdijk, Peter (2009): Du musst Dein Leben ändern. Frankfurt am Main: Suhrkamp.

Smit, Jörgen (1989): Der werdende Mensch. Zur meditativen Vertiefung des Erziehens. Stuttgart : Verlag Freies Geistesleben.

\section{Schlagworte}

Entfaltungsraum, pädagogische Architektur, Schulentwicklung, Prozessarchitektur, Lernen im Leben, Paradigmenwechsel, fachübergreifendes Lernen und Arbeiten, Zusammenhangswissen 